\title{
Mittag-Leffler-Padé approximations for the numerical solution of space and time fractional diffusion equations
}

\author{
Abdollah Borhanifar*, Sohrab Valizadeh \\ Faculty of Mathematical Sciences, University of Mohaghegh Ardabili, P.O. Box 179, Ardabil, Iran \\ *Corresponding author E-mail: borhani@uma.ac.ir
}

\begin{abstract}
Copyright (c)2015 Abdollah Borhanifar and Sohrab Valizadeh. This is an open access article distributed under the Creative Commons Attribution License, which permits unrestricted use, distribution, and reproduction in any medium, provided the original work is properly cited.
\end{abstract}

\begin{abstract}
Anomalous diffusion and non-exponential relaxation patterns can be described by a space - time fractional diffusion equation. This paper aims to present a Padé approximation for Mittag-Leffler function mixed finite difference method to develop a numerical method to obtain an approximate solution for the space and time fractional diffusion equation. The truncation error of the method is theoretically analyzed. It is proved that the numerical proposed method is unconditionally stable from the matrix analysis point of view. Finally, some numerical results are given, which demonstrate the efficiency of the approximate scheme.
\end{abstract}

Keywords: Fractional diffusion equation, Padé approximation, finite difference method, Mittag-Leffler function, stability and convergence.

\section{Introduction}

In recent years, many research studies have been focused on partial differential equations of fractional order because of their various applications in fluid mechanics, viscoelasticity, biology, physics, and engineering. Fractional calculus in mathematics is a natural extension of integer-order calculus. It has been used in modeling many physical processes arisen from real-life problems, for instance, the modeling of the transport of passive tracers carried by fluid flows in a porous medium underground water hydrology. Studies of the complicated phenomena of the interstitial fluid flows are still under intensive researches and are particularly challenging for quantitative analysis and modeling. In recent years, considerable works have been done in this area, both in theory and applications. Citing all papers and books in this area will be far too many. We cite only some few key references as instance $[1,2,3,4,5]$.

Recently, some researchers found out that the fractional model is more suitable than the integer order. Suitable mathematical models are the diffusion equations with time and/or space fractional derivatives, where the classical first order derivative in time is replaced by the Caputo fractional derivative of order $\gamma \in(0,1)$, and the second order derivative in space is essentially replaced by the Riemann-Liouville fractional derivative of order $\alpha \in(1,2]$.

Generally, it is not easy to derive analytical solutions for most of fractional partial differential equations. Therefore, it is vital to develop some reliable and efficient techniques to solve fractional partial differential equations. The numerical solution of fractional partial differential equations has attached considerable attention from many researchers. During the past decades, a plenty full of numerical schemes have been developed. These methods include finite difference approximation methods $[6,7,8,9,10,11,12]$, finite volume methods [13, 14], variational 
iteration method [15, 16], spline functions [17, 18], Tau method [19], generalized differential transform methods [20, 21], the Adomian decomposition methods [22, 23, 24] and Chebyshev spectral method [25].

We consider following space and time fractional diffusion equation in a finite domain associated with initial value and zero Dirichlet boundary conditions

$\frac{\partial^{\gamma} u(x, t)}{\partial t^{\gamma}}=D \frac{\partial^{\alpha} u(x, t)}{\partial x^{\alpha}}$,

$u(x, 0)=f(x)$,

$u(0, t)=u(L, t)=0$

The variable $u(x, t)$ represents, for example, a concentration; $D>0$ is the anomalous diffusion coeficient. The time fractional derivative $\frac{\partial^{\gamma} u(x, t)}{\partial t^{\gamma}}$ is the Caputo fractional derivative of order $\gamma(0<\gamma \leq 1)$, while the space fractional derivative $\frac{\partial^{\alpha} u(x, t)}{\partial x^{\alpha}}$ is the Riemann-Liouville fractional derivative of order $\alpha(1<\alpha \leq 2)$.

The main objective of the present article is introduce a Padé approximation method to estimate of Mittag-Leffler function, and apply this method for fractional differential equation that obtained from fractional partial differential equation via shifted Grünwald estimate.

The layout of the paper is as follows: In Section 2, a brief review of the theory of fractional calculus will be given to fix notation and provide a convenient reference. In Section 3, we construct and develop algorithms to solve space and time fractional diffusion equations by using finite difference and Padé approximation methods. In Section 4, we discuss on the stability of the proposed method. In Section 5, we give some numerical examples which confirm the obtained theoretical results. This paper ends with a conclusion in Section 6 .

\section{Preliminaries and Basic Definitions}

In this section, we give some basic definitions and properties of fractional calculus theory which shall be used in this paper:

Definition 2.1 A real function $f(x), x>0$ is said to be in the space $C_{\mu}, \mu \in \boldsymbol{R}$ if there exists a real number $p>\mu$, such that $f(x)=x^{p} g(x)$ where $g(x) \in C(0, \infty)$ and it is said to be in the space $C_{\mu}^{n}$ if and only if $f^{(n)} \in C_{\mu}, n \in \boldsymbol{N}$.

Definition 2.2 The Riemann-Liouville fractional integral operator of order $\alpha \geq 0$, of a function $f \in C_{\mu}, \mu \geq-1$, is defined as

$J^{\alpha} f(x)=\frac{1}{\Gamma(\alpha)} \int_{0}^{x}(x-t)^{\alpha-1} f(t) d t, \quad \alpha>0, \quad x>0$.

Properties of the operator $J^{\alpha}$ can be found in [4] and we mention only some following:

For $f \in C_{\mu}, \mu \geq-1, \alpha, \beta \geq 0, \gamma \geq-1$ :

$J^{0} f(x)=f(x)$,

$J^{\alpha} x^{\gamma}=\frac{\Gamma(\gamma+1)}{\Gamma(\alpha+\gamma+1)} x^{\alpha+\gamma}$

$J^{\alpha} J^{\beta} f(x)=J^{\alpha+\beta} f(x)$,

$J^{\alpha} J^{\beta} f(x)=J^{\beta} J^{\alpha} f(x)$. 
Definition 2.3 For $m$ to the smallest integer that exceeds $\alpha, n$ to the smallest integer that exceeds $\beta$, the Caputo time- fractional derivative operator of order $\alpha>0$ is defined as [3]

$D_{t}^{\alpha} u(x, t)=\frac{\partial^{\alpha} u(x, t)}{\partial t^{\alpha}}= \begin{cases}\frac{1}{\Gamma(m-\alpha)} \int_{0}^{t}(t-\tau)^{m-\alpha-1} \frac{\partial^{m} u(x, \tau)}{\partial \tau^{m}} d \tau, & m-1<\alpha<m ; \\ \frac{\partial^{m} u(x, t)}{\partial t^{m}}, & \alpha=m \in N .\end{cases}$

and the Riemann-Liouville space-fractional derivative operator of order $\beta>0$ is defined as [4]

$D_{x}^{\beta} u(x, t)=\frac{\partial^{\beta} u(x, t)}{\partial x^{\beta}}= \begin{cases}\frac{1}{\Gamma_{(n-\beta)}} \frac{\partial^{n}}{\partial x^{n}} \int_{0}^{x}(x-\tau)^{n-\beta-1} u(\tau, t) d \tau, & n-1<\beta<n ; \\ \frac{\partial^{n} u(x, t)}{\partial x^{n}}, & \beta=n \in N .\end{cases}$

Definition 2.4 For $\alpha>0$ shifted Grünwald formula defines:

$\frac{\partial^{\alpha} f(x)}{\partial x^{\alpha}}=\lim _{M \rightarrow \infty} \frac{1}{h^{\alpha}} \sum_{s=0}^{M} \omega_{s}^{(\alpha)} f(x-(k-1) h)$,

such that shifted Grünwald estimate for fractional derivative defines [26]:

$\frac{\partial^{\alpha} f(x)}{\partial x^{\alpha}}=\frac{1}{h^{\alpha}} \sum_{s=0}^{M} \omega_{s}^{(\alpha)} f(x-(k-1) h)+O\left(h^{\alpha}\right)$,

and $M$ is a positive integers and $h=\frac{x_{R}-x_{L}}{M}$. Moreover, normalized Grünwald Weights are defined by:

$\omega_{s}^{(\alpha)}=(-1)^{s} \frac{\Gamma(\alpha+1)}{\Gamma(s+1) \Gamma(\alpha-s+1)}, \quad s=0,1,2, \ldots$

Definition 2.5 The Mittag-Leffler function $E_{\alpha}(z)$ with $\alpha>0$ is defined by the following series representation, valid in the whole complex plane [27]:

$E_{\alpha}(z)=\sum_{n=0}^{\infty} \frac{z^{n}}{\Gamma(n \alpha+1)}, \quad \alpha>0, \quad z \in C$.

Interestingly, Mittag-Leffler functions are eigenfunctions of the Caputo fractional derivative of order $\gamma \leq 1$ :

$D_{t}^{\gamma} E_{\gamma}\left(\omega t^{\gamma}\right)=\frac{1}{\Gamma(1-\gamma)} \int_{0}^{t}(t-\tau)^{-\gamma} \frac{d E_{\gamma}\left(\omega \tau^{\gamma}\right)}{d \tau} d \tau=\omega E_{\gamma}\left(\omega t^{\gamma}\right)$

\section{Mittag-Leffler-Padé approximation for fractional diffusion equations}

To present the Padé approximation - finite difference method, we give some notation: $k=\Delta t$ and $h=\Delta x$ are the time and spatial step respectively, the coordinates of the mesh points are $x_{i}=a+i h, i=0,1,2, \ldots, m, m=\frac{b-a}{h}$ and $t_{n}=n k, n=0,1,2, \ldots$, and the values $u(x, t)$ at these grid points are $u\left(x_{i}, t_{n}\right) \equiv u_{i, n}$.

\subsection{Spatial approximations}

Assume that $u(x, t)$ is twice differentiable with respect to $x$ and replace the space fractional partial derivatives in (1) with respect to $x$ by the shifted Grünwald estimate:

$\left.\frac{\partial^{\alpha} u(x, t)}{\partial x^{\alpha}}\right|_{\left(x_{i}, t\right)}=\frac{1}{h^{\alpha}} \sum_{s=0}^{i+1} \omega_{s}^{(\alpha)} u\left(x_{i-s+1}, t\right)+O\left(h^{\alpha}\right), \quad$ as $\quad h \rightarrow 0$,

which can be derived by using the fractional Taylor series expansion of $u\left(x_{i}+h, t\right), u\left(x_{i}-h, t\right), \ldots, u(a, t)$. This discretization results in an initial-value fractional problem of the form:

$\frac{d^{\gamma} U(t)}{d t^{\gamma}}=A U(t), \quad U(0)=U_{0}$,

where the matrix $A$ is as follows: 


$$
A_{i, j}=\frac{1}{h^{\alpha}} \begin{cases}0, & \text { when } j>i+1 ; \\ \omega_{1}^{(\alpha)}, & \text { when } j=i ; \\ \omega_{i-j+1}^{(\alpha)}, & \text { otherwise. }\end{cases}
$$

By using (5), the exact solution of (7) can be written as

$U(t)=U_{0} E_{\gamma}\left(t^{\gamma} A\right)$,

and we have

$U(k)=U_{0} E_{\gamma}\left(k^{\gamma} A\right)$.

By repeat above process for the next time step, we have

$U(2 k)=U(k) E_{\gamma}\left(k^{\gamma} A\right)$.

Therefore, exact solution satisfies the recurrence formula:

$U\left(t_{n+1}\right)=E_{\gamma}\left(k^{\gamma} A\right) U\left(t_{n}\right)$.

Recurrence formula (8) is the basis of different time stepping schemes that depond on how we approximate the matrix Mittag-Leffler function. we shall use positivity preserving Padé approximations of matrix Mittag-Leffler functions $E_{\gamma}\left(k^{\gamma} A\right), 0<\gamma \leq 1$ to construct a family of time stepping schemes. the special case for $\gamma=1$ has been worked for the numerical solution of Burgers equation in the [28].

\subsection{The Padé approximation to $E_{\gamma}(\theta), \theta$ real}

Assume that $E_{\gamma}(\theta)$ is approximated by $\frac{1}{1+q_{1} \theta+q_{2} \theta^{2}}$, where $q_{1}$ and $q_{2}$ are constants. Determination of $q_{1}$ and $q_{2}$ requires two equations, which will come from the coefficients of $\theta$ and $\theta^{2}$. So, the leading error term will be order $\theta^{3}$. Hence

$E_{\gamma}(\theta) \equiv \frac{1}{1+q_{1} \theta+q_{2} \theta^{2}}+c_{3} \theta^{3}+c_{4} \theta^{4}+\ldots$.

Therefore,

$$
\begin{aligned}
\left(1+q_{1} \theta+q_{2} \theta^{2}\right)(1+ & \left.\frac{\theta}{\Gamma(\gamma+1)}+\frac{\theta^{2}}{\Gamma(2 \gamma+1)}+\frac{\theta^{3}}{\Gamma(3 \gamma+1)}+\ldots\right) \\
& =1+\left(1+q_{1} \theta+q_{2} \theta^{2}\right)\left(c_{3} \theta^{3}+c_{4} \theta^{4}+\ldots\right)
\end{aligned}
$$

Or

$$
\left(q_{1}+\frac{1}{\Gamma(\gamma+1)}\right) \theta+\left(q_{2}+\frac{q_{1}}{\Gamma(\gamma+1)}+\frac{1}{\Gamma(2 \gamma+1)}\right) \theta^{2}+
$$

$\left(c_{3}+\frac{q_{2}}{\Gamma(\gamma+1)}+\frac{q_{1}}{\Gamma(2 \gamma+1)}+\frac{1}{\Gamma(3 \gamma+1)}\right) \theta^{3}+$ higher order term $\equiv 0$.

This is satisfied uniquely to term of order three by

$q_{1}=-\frac{1}{\Gamma(\gamma+1)}$

$q_{2}=\frac{1}{\Gamma(\gamma+1)^{2}}-\frac{1}{\Gamma(2 \gamma+1)}$

and

$c_{3}=\frac{1}{\Gamma(3 \gamma+1)}-\frac{2}{\Gamma(\gamma+1) \Gamma(2 \gamma+1)}+\frac{1}{\Gamma(\gamma+1)^{3}}$.

The rational approximation

$\frac{1}{1-\frac{1}{\Gamma(\gamma+1)} \theta+\left(\frac{1}{\Gamma(\gamma+1)^{2}}-\frac{1}{\Gamma(2 \gamma+1)}\right) \theta^{2}}$, 
is called the $[2,0]$ Padé approximation of order 2 to $E_{\gamma}(\theta)$ and has a leading error term of order 3 .

Generally, it is possible to approximate $E_{\gamma}(\theta)$ by

$E_{\gamma}(\theta)=\frac{1+p_{1} \theta+p_{2} \theta^{2}+\ldots+p_{T} \theta^{T}}{1+q_{1} \theta+q_{2} \theta^{2}+\ldots+q_{S} \theta^{S}}+c_{S+T+1} \theta^{S+T+1}+O\left(\theta^{S+T+2}\right)$,

where $c_{S+T+1}$ is an independent constant. The rational function

$R_{S, T}(\theta)=\frac{1+p_{1} \theta+p_{2} \theta^{2}+\ldots+p_{T} \theta^{T}}{1+q_{1} \theta+q_{2} \theta^{2}+\ldots+q_{S} \theta^{S}}=\frac{P_{T}(\theta)}{Q_{S}(\theta)}$

is called the $[S, T]$ Padé approximant of order $(S+T)$ to $E_{\gamma}(\theta)$.

\subsection{Padé Rational Approximation Algorithm}

Here we present an algorithm that gives the Padé approximants to $E_{\gamma}(),. 0<\gamma \leq 1$.

To obtain the rational approximation

$r(x)=\frac{p(x)}{q(x)}=\frac{\sum_{i=0}^{n} p_{i} x^{i}}{\sum_{j=0}^{m} q_{j} x^{j}}$

for a Mittag-Leffler function $\left(E_{\gamma}(x), 0<\gamma \leq 1\right)$ we use following algorithm [29]:

Algorithm:

Input: Nonnegative integers $m$ and $n$.

Output: Coefficients $q_{0}, q_{1}, \ldots, q_{m}$ and $p_{0}, p_{1}, \ldots, p_{n}$.

step 1: Set $\mathrm{N}=\mathrm{m}+\mathrm{n}$.

step 2: For $i=0,1, \ldots, N$ set $a_{i}=\frac{1}{\Gamma(i \gamma+1)}$.

step 3: Set $q_{0}=p_{0}=1$.

step 4: For $i=0,1, \ldots, N$ do steps 5 -10. (Set up a linear system with matrix $B$.)

step 5: For $j=0,1, \ldots, i-1$ if $j \leq n$, then set $b_{i, j}=0$.

step 6: For $i \leq n$, then set $b_{i, i}=0$.

step 7: For $j=i+1, i+2, \ldots, N$ if $j \leq N$, then set $b_{i, j}=0$.

step 8: For $j=1,2, \ldots, i$ if $j \leq m$, then set $b_{i, n+j}=-a_{i-j}$.

step 9: For $j=n+i+1, n+i+2, \ldots, N$ set $b_{i, j}=0$.

step 10: Set $b_{i, N+1}=a_{i}$. (Steps 11-22 solve the linear system using partial pivoting.)

step 11: For $i=n+1, n+2, \ldots, N-1$ do steps 12-18.

step 12: Let $k$ be smallest integer with $i \leq k \leq N$ and $\left|b_{k, i}\right|=\max _{i \leq j \leq N}\left|b_{j, i}\right|$. (Find pivot element.)

step 13: If $b_{k, i}=0$, then OUTPUT ("The system is singular"); Stop.

step 14: If $k \neq i$, then (Interchange row $i$ and row $k$.) for $j=i, i+1, \ldots N+1$ set $b_{C O P Y}=b_{i, j} ; b_{i, j}=b_{k, j}$; $b_{k, j}=b_{C O P Y}$.

step 15: For $j=i+1, i+2, \ldots, N$ do steps 16-18. (Perform elimination.)

step 16: Set $x m=\frac{b_{j, i}}{b_{i, i}}$.

step 17: For $k=i+1, i+2, \ldots, N+1$ set $b_{j, k}=b_{j, k}-x m . b_{i, k}$.

step 18: Set $b_{j, i}=0$.

step 19: If $b_{N, N}=0$, then OUTPUT ("The system is singular"); Stop.

step 20: If $m>0$, then set $q_{m}=\frac{b_{N, N+1}}{b_{N, N}}$. (Start backward substitution.)

step 21: For $i=N-1, N-2, \ldots, n+1$ set $q_{i-n}=\frac{b_{i, N+1}-\sum_{j=i+1}^{N} b_{i, j} q_{j-n}}{b_{i, i}}$.

step 22: For $i=n, n-1, \ldots, 1$ set $p_{i}=b_{i, N+1}-\sum_{j=n+1}^{N} b_{i, j} q_{j-n}$.

step 23: OUTPUT $\left(q_{0}, q_{1}, \ldots, q_{m}, p_{0}, p_{1}, \ldots, p_{n}\right)$; Stop. (The procedure was successful.)

By using the above-mentioned algorithm, we have constructed four L-stable numerical schemes using $R_{m, 0}\left(k^{\gamma} A\right)$ for $m=1,2,3$ and 4 :

(a) A first order scheme (Backward Euler):

$U\left(t_{n+1}\right)=\left(I+q_{1} k^{\gamma} A\right)^{-1} U\left(t_{n}\right)$,

(b) A second order scheme:

$U\left(t_{n+1}\right)=\left(I+q_{1} k^{\gamma} A+q_{2} k^{2 \gamma} A^{2}\right)^{-1} U\left(t_{n}\right)$, 
(c) A third order scheme:

$U\left(t_{n+1}\right)=\left(I+q_{1} k^{\gamma} A+q_{2} k^{2 \gamma} A^{2}+q_{3} k^{3 \gamma} A^{3}\right)^{-1} U\left(t_{n}\right)$,

(d) A fourth order scheme:

$U\left(t_{n+1}\right)=\left(I+q_{1} k^{\gamma} A+q_{2} k^{2 \gamma} A^{2}+q_{3} k^{3 \gamma} A^{3}+q_{4} k^{4 \gamma} A^{4}\right)^{-1} U\left(t_{n}\right)$,

where

$q_{1}=-\frac{1}{\Gamma(\gamma+1)}$,

$q_{2}=\frac{1}{\Gamma(\gamma+1)^{2}}-\frac{1}{\Gamma(2 \gamma+1)}$,

$q_{3}=-\frac{1}{\Gamma(\gamma+1)^{3}}+\frac{2}{\Gamma(\gamma+1) \Gamma(2 \gamma+1)}-\frac{1}{\Gamma(3 \gamma+1)}$,

and

$q_{4}=\frac{1}{\Gamma(\gamma+1)^{4}}-\frac{3}{\Gamma(\gamma+1)^{2} \Gamma(2 \gamma+1)}+\frac{2}{\Gamma(\gamma+1) \Gamma(3 \gamma+1)}+\frac{1}{\Gamma(2 \gamma+1)^{2}}-\frac{1}{\Gamma(4 \gamma+1)}$.

\section{Accuracy and stability analysis}

In this section we prove the convergence of the numerical methods by showing that they are consistent and stable. First, we start to study the consistency of the numerical methods and then we present the stability result.

Theorem 4.1 The truncation error of the difference schemes (9), (10), (11) and (12) are $O\left(h^{\alpha}+k^{2 \gamma}\right), O\left(h^{\alpha}+k^{3 \gamma}\right)$, $O\left(h^{\alpha}+k^{4 \gamma}\right)$ and $O\left(h^{\alpha}+k^{5 \gamma}\right)$, respectively.

Proof. It has been shown that the order of shifted Grünwald estimate to approximate the Riemann-Liouville spacefractional derivative is equal to order of fractional derivative [26]. In addition, for Padé approximation scheme to approximate $E_{\gamma}(\theta)$ we have

$E_{\gamma}(\theta)=\frac{1+p_{1} \theta+p_{2} \theta^{2}+\ldots+p_{T} \theta^{T}}{1+q_{1} \theta+q_{2} \theta^{2}+\ldots+q_{S} \theta^{S}}+O\left(\theta^{S+T+1}\right)$.

By substitution $\theta=k^{\gamma} A, T=0$ and $S=1,2,3,4$, we see that the order of Padé approximation schemes $[1,0],[2,0]$, $[3,0]$ and $[4,0]$ to approximate $E_{\gamma}\left(k^{\gamma} A\right)$ are $O\left(k^{2 \gamma}\right), O\left(k^{3 \gamma}\right), O\left(k^{4 \gamma}\right)$ and $O\left(k^{5 \gamma}\right)$, respectively.

Therefore, the above explanation demonstrate that the truncation error of the difference schemes (9), (10), (11) and (12) are $O\left(h^{\alpha}+k^{2 \gamma}\right), O\left(h^{\alpha}+k^{3 \gamma}\right), O\left(h^{\alpha}+k^{4 \gamma}\right)$ and $O\left(h^{\alpha}+k^{5 \gamma}\right)$, respectively.

Lemma 4.2 (Gerschgorin)[10, 30] Let the matrix $A \equiv\left(a_{i, j}\right)$ has eigenvalues $\lambda$ and define the absolute row and column sums by

$r_{i} \equiv \sum_{j=1, j \neq i}^{n}\left|a_{i, j}\right|, c_{j} \equiv \sum_{i=1, i \neq j}^{n}\left|a_{i, j}\right|$,

then,

(a) Each eigenvalues lies in the union of the row circles $R_{i}, i=1,2, \ldots, n$ where

$R_{i} \equiv\left\{z:\left|z-a_{i, i}\right| \leq r_{i}\right\}$

(b) Each eigenvalues lies in the union of the column circles $C_{j}, j=1,2, \ldots, n$ where

$C_{j} \equiv\left\{z:\left|z-a_{j, j}\right| \leq c_{j}\right\}$.

Theorem 4.3 Numerical algorithms (9), (10), (11) and (12) for the problem (1)-(3) are unconditionally stable. 
Proof. Since $\omega_{1}^{(\alpha)}, 0<\alpha \leq 1$ and for $i \neq 1$, we have $\omega_{i}^{(\alpha)} \geq 0$ (the strictly inequality holds for non-integer values of $\alpha$ ). We also have $\sum_{k=0, k \neq 1}^{M} \omega_{k}^{(\alpha)} \leq-\omega_{1}^{(\alpha)}$, which follows from the well-known equality $\sum_{k=0}^{\infty} \omega_{k}^{(\alpha)}=0$. According to the Gerschgorin theorem the eigenvalues of matrix $A$ lie in the union of the $M$ row circles centered at $A_{i, i}$, with radius: $r_{i}=\sum_{k=0, k \neq i}^{M}\left|A_{i, k}\right|$. Now from definition of $A$ we have:

$A_{i, i}=\omega_{1}^{(\alpha)}=-\alpha$

and

$r_{i}=\sum_{k=0, k \neq i}^{M}\left|A_{i, k}\right|=\sum_{k=0, k \neq i}^{i+1}\left|A_{i, k}\right|=\sum_{k=0, k \neq i}^{i+1}\left|\omega_{i-k+1}^{(\alpha)}\right|=\sum_{k=0, k \neq i}^{i+1} \omega_{i-k+1}^{(\alpha)} \leq-\omega_{1}^{(\alpha)}=\alpha ;$

and also in the union of the $M$ column circles centered at $A_{j, j}$, with radius: $c_{j}=\sum_{k=0, k \neq j}^{M}\left|A_{k, j}\right|$.

By above mentioned similarly process we have:

$A_{j, j}=\omega_{1}^{(\alpha)}=-\alpha ;$

and

$c_{j}=\sum_{k=0, k \neq j}^{M}\left|A_{k, j}\right|=\sum_{k=0, k \neq j}^{j+1}\left|A_{k, j}\right|=\sum_{k=0, k \neq j}^{j+1}\left|\omega_{k-j+1}^{(\alpha)}\right|=\sum_{k=0, k \neq i}^{i+1} \omega_{k-j+1}^{(\alpha)} \leq-\omega_{1}^{(\alpha)}=\alpha$.

Hence, we proved that these row and column Gerschgorin disks are within the left half of the complex plane. In addition, since following fractional transformations map the left half of the complex plane onto inner unit disks that associated to (9), (10), (11) and (12), respectively

$\pi_{j}(z)=\frac{1}{1+\sum_{i=1}^{j} q_{i} k^{i \gamma} z^{i}}, \quad j=1,2,3,4$.

where $q_{i}$ for $i=1,2,3,4$ are coefficient that refer to equations (9), (10), (11) and (12), we have if $\lambda$ be the eigenvalue of the matrix $A$, then

$\left|\pi_{j}(z)\right|<1, \quad j=1,2,3,4$.

So, Numerical algorithms (9), (10), (11) and (12) are unconditionally stable.

\section{Numerical Results}

To demonstrate the efficiency of the proposed methods in the present paper, two test examples are carried out. We implemented proposed schemes for numerical approximation of the space and time fractional diffusion equations with MATLAB 7.10 software. We also use our solution method to illustrate the changes in solution behavior that arise when the exponent is varied from integer order to fractional order.

Example 5.1 Consider the following space and time fractional diffusion equation:

$\frac{\partial^{\gamma} u(x, t)}{\partial t^{\gamma}}=\frac{\partial^{\alpha} u(x, t)}{\partial x^{\alpha}}, \quad 0<\gamma \leq 1, \quad 1<\alpha \leq 2, \quad(x, t) \in[0,1] \times[0, T]$,

with initial value and zero Dirichlet boundary conditions:

$u(x, 0)=x(1-x), \quad 0 \leq x \leq 1$,

$u(0, t)=u(L, t)=0, \quad 0 \leq t \leq T$

Analytic solution of Eqs. (13)-(15) for $\alpha=2$ and $\gamma=1$ is given by

$u(x, t)=\sum_{n=1}^{\infty} \frac{4}{(n \pi)^{3}}\left[(-1)^{n+1}+1\right] \sin (n \pi x) e^{-(n \pi)^{2} t}$, 


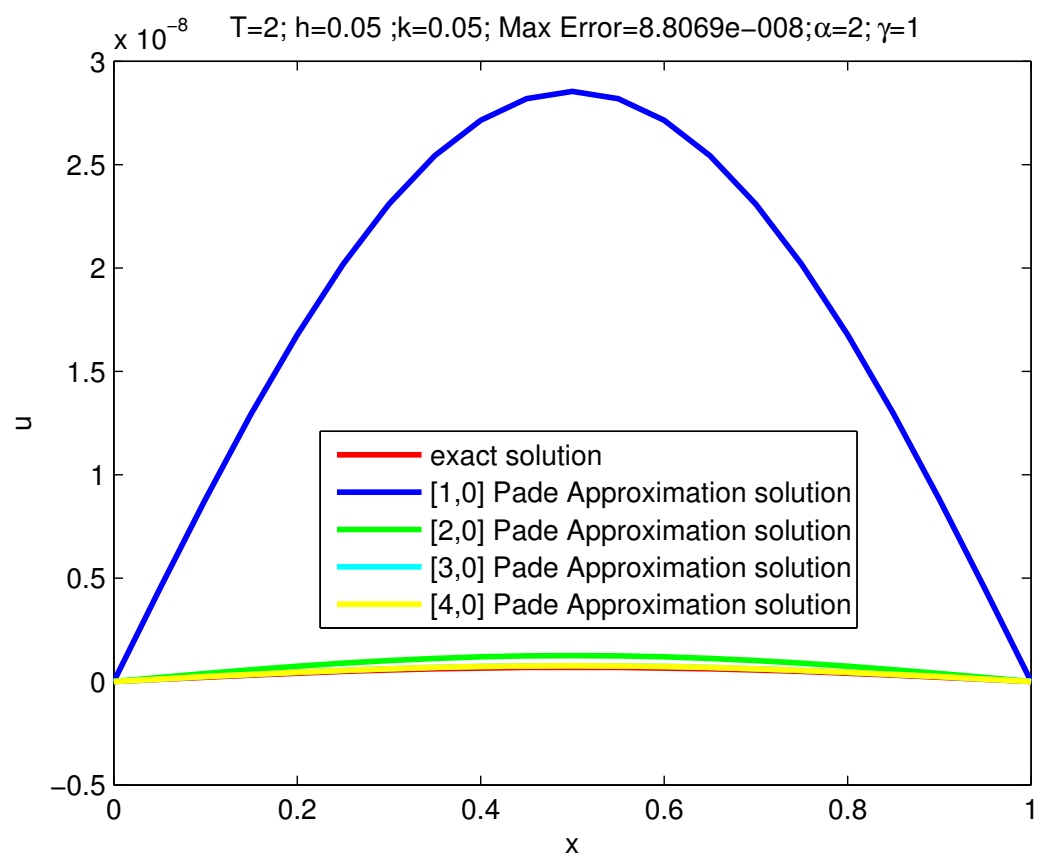

Figure 1: Comparison of the numerical solutions using the proposed methods (9)-(12) with exact solution for the problem (13)-(15) for $\alpha=2, \gamma=1, h=0.05$ and $k=0.05$, at $T=2$.

Table 1: Absolute error for Mittag - Leffler Padé Approximation and finite difference method in example 1

\begin{tabular}{cccccc}
\hline$h=\Delta x$ & $k=\Delta t$ & {$[1,0]$ Padé Appr. } & {$[2,0]$ Padé Appr. } & {$[3,0]$ Padé Appr. } & {$[4,0]$ Padé Appr. } \\
\hline 0.1 & 0.05 & $6.7679 \mathrm{e}-008$ & $1.5943 \mathrm{e}-009$ & $3.9272 \mathrm{e}-010$ & $2.8240 \mathrm{e}-010$ \\
0.1 & 0.01 & $2.9191 \mathrm{e}-009$ & $3.2452 \mathrm{e}-010$ & $2.7231 \mathrm{e}-010$ & $2.7105 \mathrm{e}-010$ \\
0.1 & 0.001 & $4.5225 \mathrm{e}-010$ & $2.7159 \mathrm{e}-010$ & $2.7103 \mathrm{e}-010$ & $2.7102 \mathrm{e}-010$ \\
0.05 & 0.05 & $8.8069 \mathrm{e}-008$ & $1.7839 \mathrm{e}-009$ & $2.4634 \mathrm{e}-010$ & $1.0671 \mathrm{e}-010$ \\
0.05 & 0.01 & $3.4687 \mathrm{e}-009$ & $1.5859 \mathrm{e}-010$ & $9.1956 \mathrm{e}-011$ & $9.0139 \mathrm{e}-011$ \\
0.05 & 0.001 & $3.2031 \mathrm{e}-010$ & $9.1053 \mathrm{e}-011$ & $9.0336 \mathrm{e}-011$ & $9.0334 \mathrm{e}-011$ \\
\hline
\end{tabular}

Figs 1, 2 and 3 show graphs of the exact solution and numerical solutions using four schemes for $\alpha=2$ and $\gamma=1$. It is clear that with the same parameter values, numerical solutions approach to the exact solution as we use higher and higher order schemes.

Error of the numerical solution $U_{n}$ is defined by

$E_{2}=\left(\sum_{i=1}^{M}\left[u_{i, n}-U_{i, n}\right]^{2}\right)^{\frac{1}{2}}$,

where $u_{n}$ is exact solution.

From Table 1, It can be seen that the numerical solutions are enough close to the exact solution for higher order schemes.

The time evolution graphs of the numerical solution of Eqs. (13)-(15) that obtained via [4,0] Padé approximation method are shown in Fig 4 for different values of $\alpha$ and $\gamma$. From Fig. 4, for fix $\gamma=1$ and $\gamma=0.5$, Convergence to zero of the fractional diffusion of order $\alpha=1.8$ is slower than the standard diffusion $(\alpha=2)$.

For fix $\alpha=2$ and $\alpha=1.8$, Convergence to zero of the fractional dispersion of order $\gamma=0.5$ is faster than standard dispersion $(\gamma=1)$.

To demonstrate the impact of proposed method to the solution behavior, another example of the space and time fractional diffusion equation with a different initial condition is now considered. 


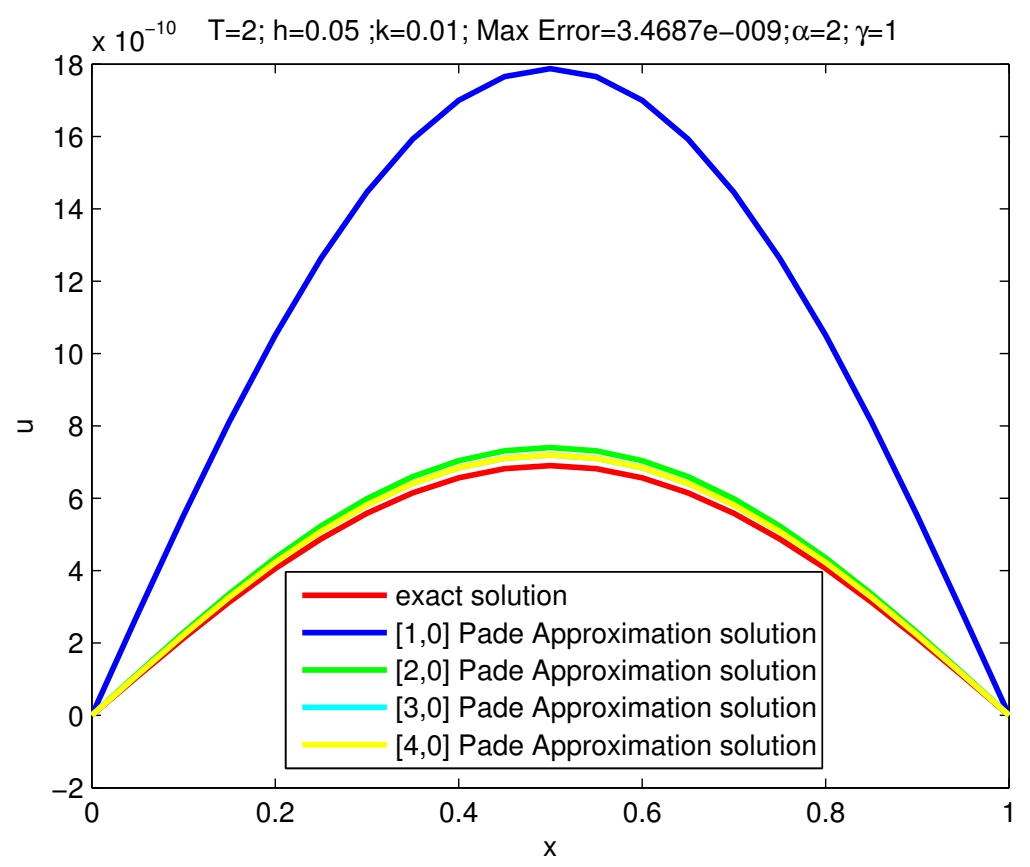

Figure 2: Comparison of the numerical solutions using the proposed methods (9)-(12) with exact solution for the problem (13)-(15) for $\alpha=2, \gamma=1, h=0.05$ and $k=0.01$, at $T=2$.

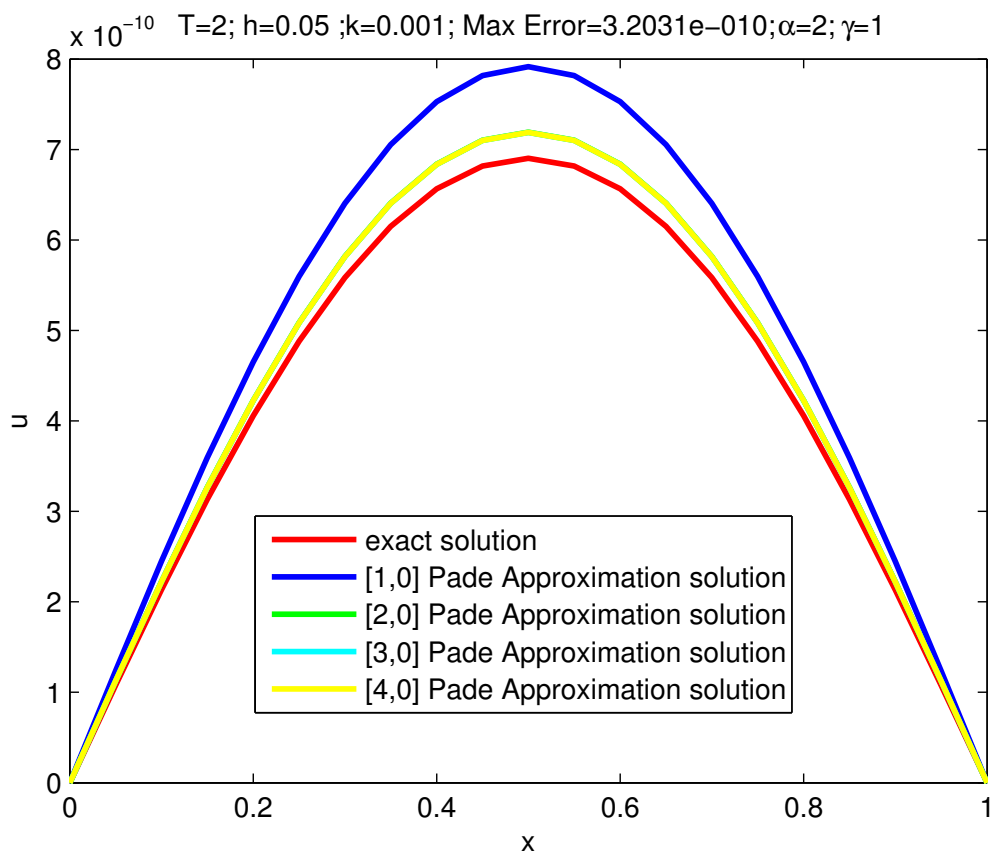

Figure 3: Comparison of the numerical solutions using the proposed methods (9)-(12) with exact solution for the problem (13)-(15) for $\alpha=2, \gamma=1, h=0.05$ and $k=0.001$, at $T=2$. 


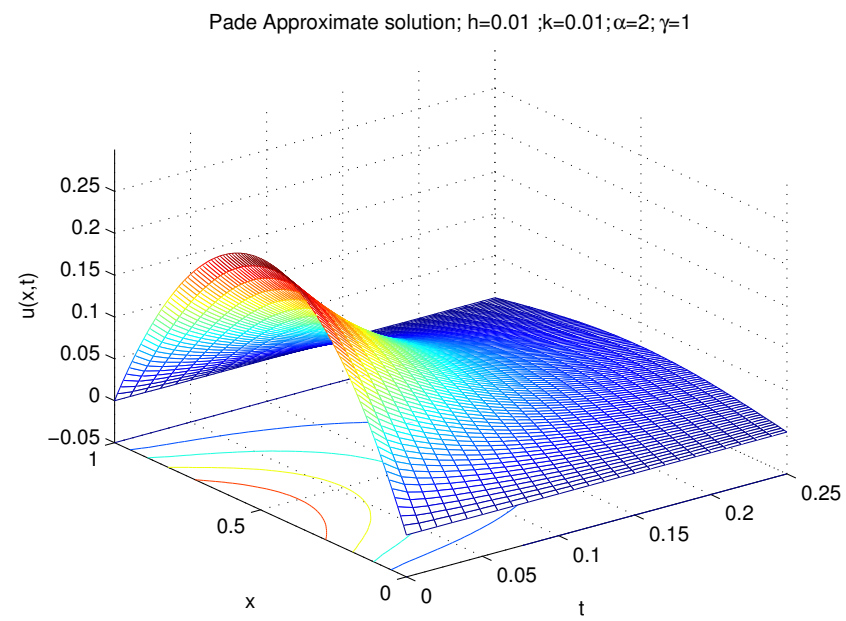

Pade Approximate solution; $\mathrm{h}=0.01 ; \mathrm{k}=0.01 ; \alpha=2 ; \gamma=0.5$

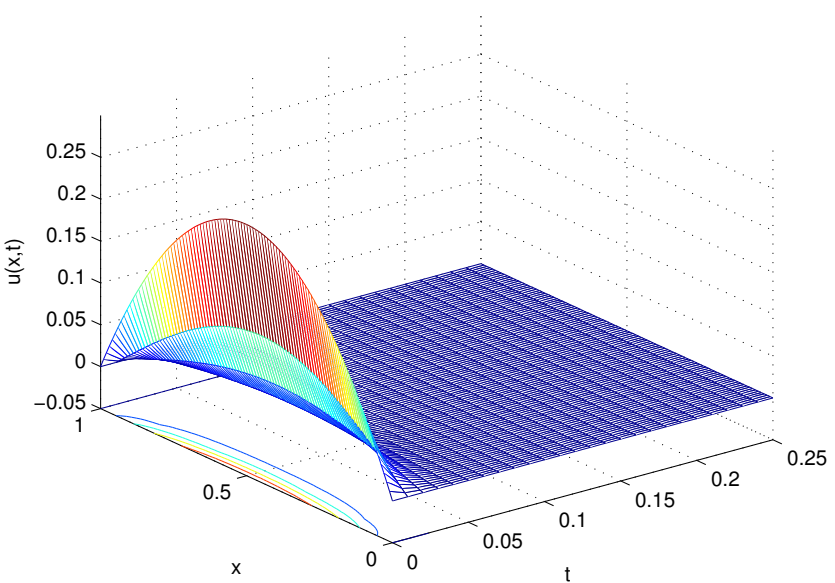

Pade Approximate solution; $\mathrm{h}=0.01 ; \mathrm{k}=0.01 ; \alpha=1.8 ; \gamma=1$

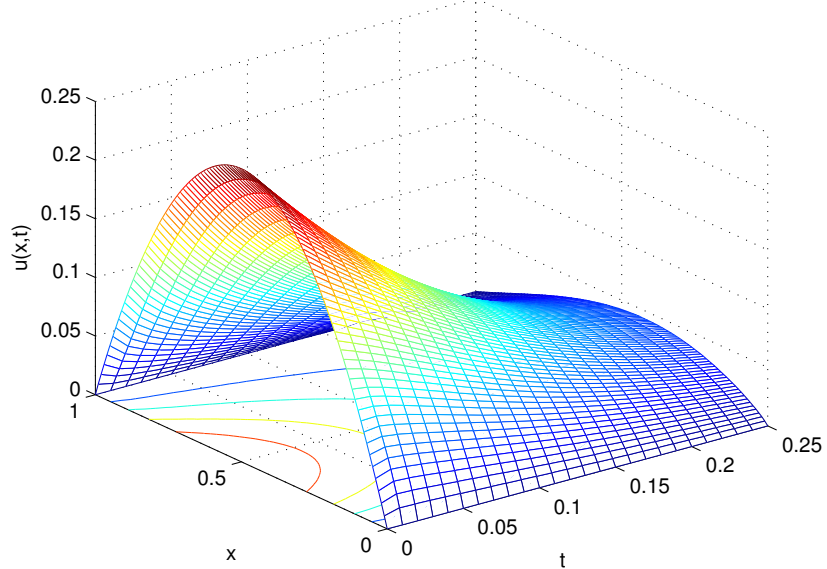

a)

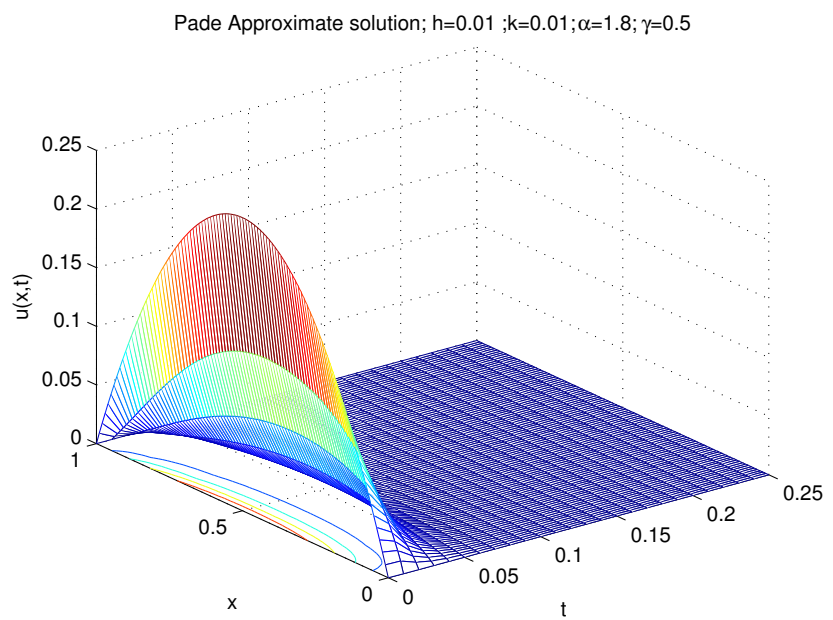

b)

d)

Figure 4: Numerical estimate of $u(x, t)$ via [4,0] Padé approximation method for the problem (13)-(15) when $t=0.25$.

Example 5.2 Consider the following space and time fractional diffusion equation:

$\frac{\partial^{\gamma} u(x, t)}{\partial t^{\gamma}}=\frac{\partial^{\alpha} u(x, t)}{\partial x^{\alpha}}, \quad 0<\gamma \leq 1, \quad 1<\alpha \leq 2, \quad(x, t) \in[0,1] \times[0, T]$,

with initial value and zero Dirichlet boundary conditions:

$u(x, 0)=\sin (\pi x), \quad 0 \leq x \leq 1$,

$u(0, t)=u(L, t)=0, \quad 0 \leq t \leq T$

Analytic solution of Eqs. (16)-(18) for $\alpha=2$ and $\gamma=1$ is given by

$u(x, t)=\sin (\pi x) e^{-\pi^{2} t}$.

Figs 5, 6 and 7 show graphs of the exact solution and numerical solutions using four schemes for $\alpha=2$ and $\gamma=1$. It is clear that with the same parameter values, numerical solutions approach to the exact solution as we use higher and higher order schemes.

From Table 2, it can be seen that $[1,0],[2,0],[3,0]$ and $[4,0]$ Padé approximation methods are stable and convergence for solving the problem (16)-(18) with $\alpha=2$ and $\gamma=1$.

The time evolution graphs of the numerical solution of Eqs. (16)-(18) that obtained via [3,0] Padé approximation method are shown in Fig. 8 for different values of $\alpha$ and $\gamma$. From Fig. 8, for fix $\gamma=1$ and $\gamma=0.5$, Convergence to 


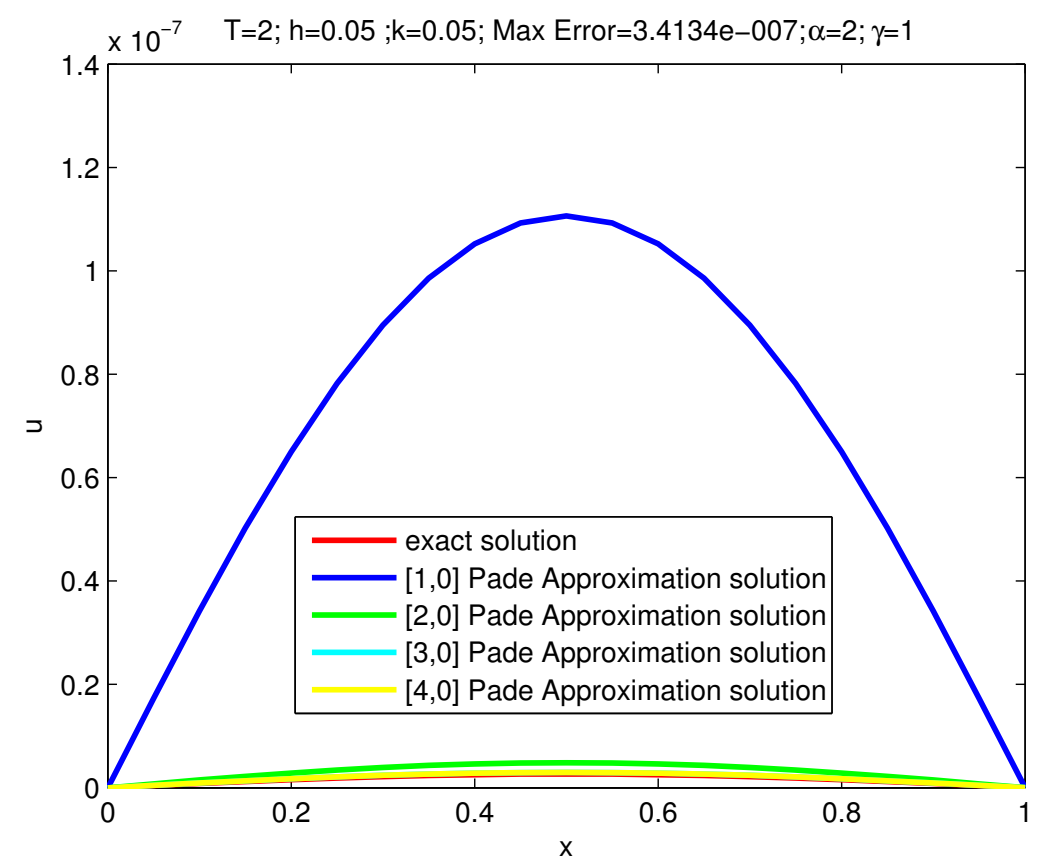

Figure 5: Comparison of the numerical solutions using the proposed methods (9)-(12) with exact solution for the problem (16)-(18) for $\alpha=2, \gamma=1, h=0.05$ and $k=0.05$, at $T=2$.

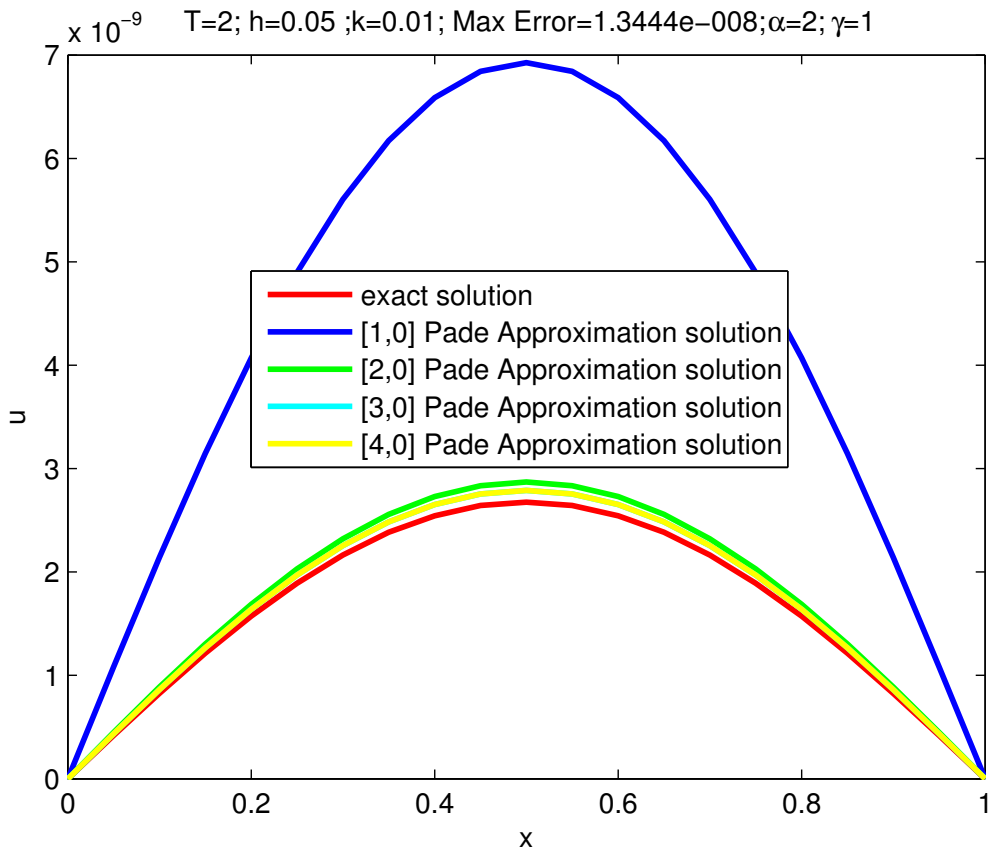

Figure 6: Comparison of the numerical solutions using the proposed methods (9)-(12) with exact solution for the problem (16)-(18) for $\alpha=2, \gamma=1, h=0.05$ and $k=0.01$, at $T=2$. 


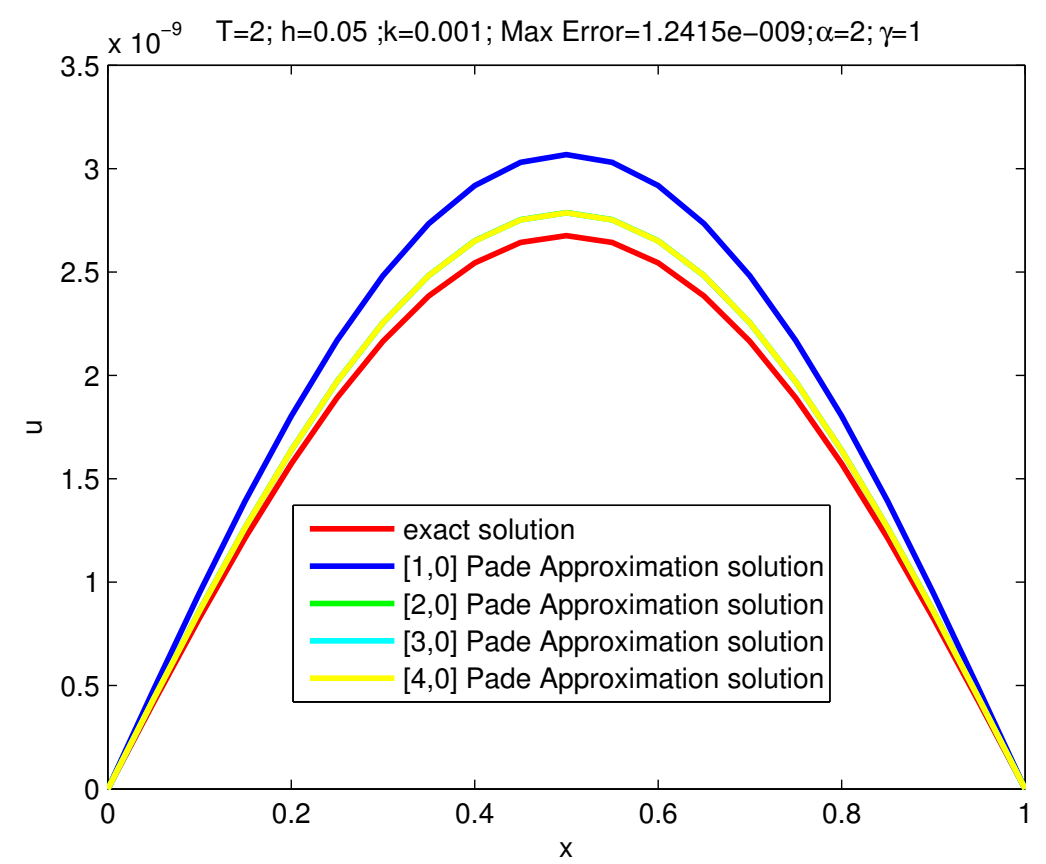

Figure 7: Comparison of the numerical solutions using the proposed methods (9)-(12) with exact solution for the problem (16)-(18) for $\alpha=2, \gamma=1, h=0.05$ and $k=0.001$, at $T=2$.

Table 2: Absolute error for Mittag - Leffler Padé Approximation and finite difference method in example 2

\begin{tabular}{cccccc}
\hline$h=\Delta x$ & $k=\Delta t$ & {$[1,0]$ Padé Appr. } & {$[2,0]$ Padé Appr. } & {$[3,0]$ Padé Appr. } & {$[4,0]$ Padé Appr. } \\
\hline 0.1 & 0.05 & $2.6232 \mathrm{e}-007$ & $6.1797 \mathrm{e}-009$ & $1.5224 \mathrm{e}-009$ & $1.0949 \mathrm{e}-009$ \\
0.1 & 0.01 & $1.1314 \mathrm{e}-008$ & $1.2581 \mathrm{e}-009$ & $1.0557 \mathrm{e}-009$ & $1.0508 \mathrm{e}-009$ \\
0.1 & 0.001 & $1.7531 \mathrm{e}-009$ & $1.0529 \mathrm{e}-009$ & $1.0507 \mathrm{e}-009$ & $1.0507 \mathrm{e}-009$ \\
0.05 & 0.05 & $3.4134 \mathrm{e}-007$ & $6.9143 \mathrm{e}-009$ & $9.5244 \mathrm{e}-010$ & $4.0549 \mathrm{e}-010$ \\
0.05 & 0.01 & $1.3444 \mathrm{e}-008$ & $6.1468 \mathrm{e}-010$ & $3.5636 \mathrm{e}-010$ & $3.4994 \mathrm{e}-010$ \\
0.05 & 0.001 & $1.2415 \mathrm{e}-009$ & $3.5292 \mathrm{e}-010$ & $3.5014 \mathrm{e}-010$ & $3.5014 \mathrm{e}-010$ \\
\hline
\end{tabular}


zero of the fractional diffusion of order $\alpha=1.8$ is slower than convergence to zero of the standard diffusion $(\alpha=2)$. For fix $\alpha=2$ and $\alpha=1.8$, Convergence to zero of the fractional dispersion of order $\gamma=0.5$ is faster than the standard dispersion $(\gamma=1)$.
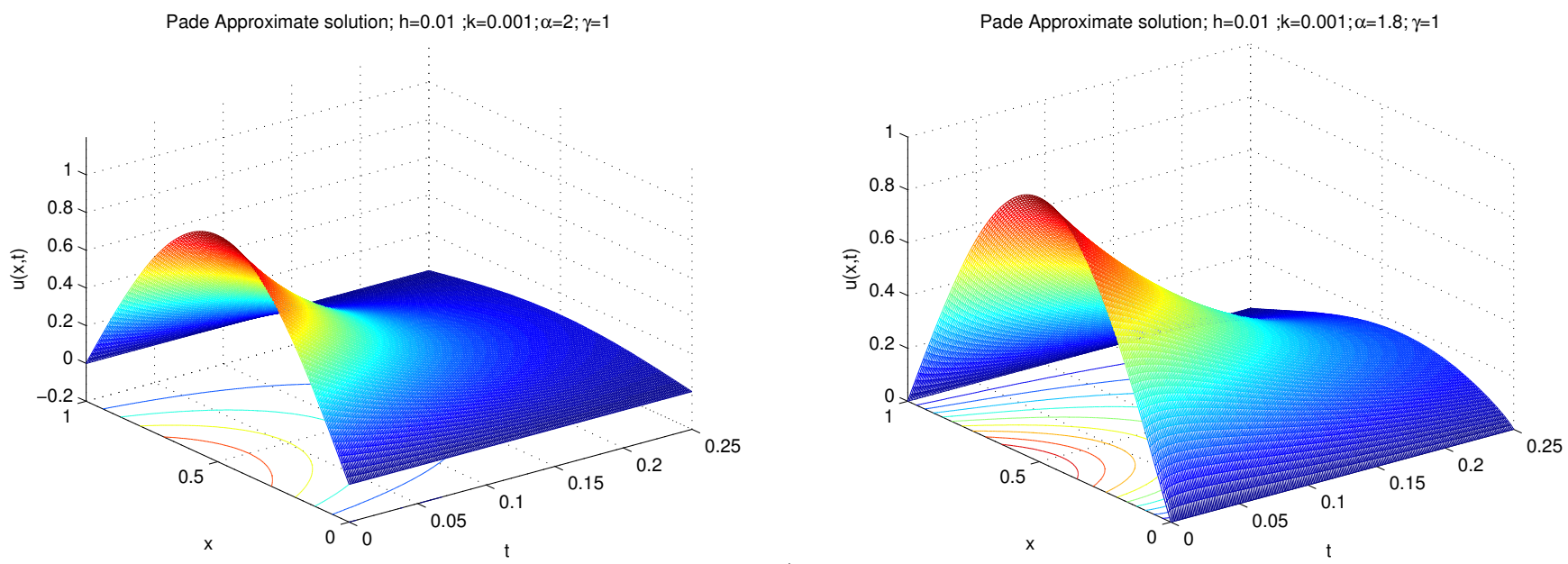

a)

Pade Approximate solution; $\mathrm{h}=0.01 ; \mathrm{k}=0.001 ; \alpha=2 ; \gamma=0.5$

Pade Approximate solution; $\mathrm{h}=0.01 ; \mathrm{k}=0.001 ; \alpha=1.8 ; \gamma=0.5$
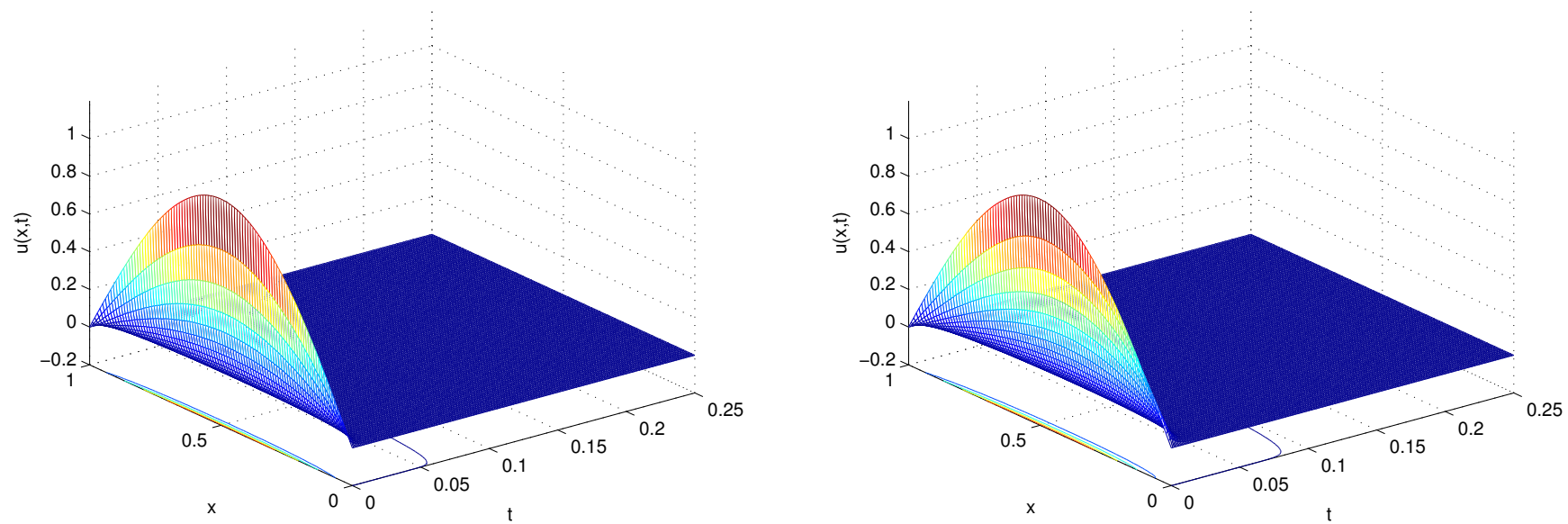

c)

Figure 8: The numerical estimate of $u(x, t)$ via [3,0] Padé approximation method for the problem $(16)-(18)$ when $t=0.25$.

\section{Conclusions}

In this paper, several numerical schemes with $2 \gamma, 3 \gamma, 4 \gamma$ and $5 \gamma$ order temporal accuracy, $0<\gamma \leq 1$, and $\alpha$ order spatial accuracy, $1<\alpha \leq 2$, to solve a space and time fractional diffusion equation with initial value and zero Dirichlet boundary conditions has been planned. The truncation error and stability of proposed methods are described by the matrix analysis method. The numerical experiment extremely consistent with our theoretical results.

\section{Acknowledgements}

This work is partially supported by Grant-in-Aid from the University of Mohaghegh Ardabili, Ardabil, Iran.

\section{References}

[1] R. Hilfer, Applications of Fractional Calculus in Physics, World Scientific, Singapore, 2000. 
[2] F. M. Atici, P. W. Eloe, Gronwall's inequality on discrete fractional calculus, Computers and Mathematics with Applications, 64 (2012) 3193-3200.

[3] A. Kilbas, H. Srivastava, J. Trujillo, Theory and Applications of Fractional Differential Equations, Elsevier, Amsterdam, 2006.

[4] I. Podlubny, Fractional Differential Equations, Academic Press, New York, 1999.

[5] I. Podlubny, Geometric and physical interpretation of fractional integration and fractional differentiation, Fractional Calculus and Applied Analysis, 5 (2002) 367-386.

[6] D. A. Murio, Implicit finite difference approximation for time fractional diffusion equations, Computers and Mathematics with Applications, 56 (2008) 1138-1145.

[7] M. M. Meerschaert, C. Tadjeran, Finite difference approximations for fractional advection-dispersion flow equations, Journal of Computational and Applied Mathematics, 172 (2004) 65-77.

[8] M. M. Meerschaert, C. Tadjeran, Finite difference approximations for two-sided space-fractional partial differential equations, Applied Numerical Mathematics, 56 (2006) 80-90.

[9] Q. Yang, F. Liu, I. Turner, Numerical methods for fractional partial differential equations with Riesz space fractional derivatives, Applied Mathematical Modelling, 34 (2010) 200-218.

[10] N.H. Sweilam, M.M. Khader, A.M. Nagy, Numerical solution of two-sided space-fractional wave equation using finite difference method, Journal of Computational and Applied Mathematics, 235 (2011) 2832-2841.

[11] N. H. Swellam, M. M. Khader, A. M. S. Mahdy, Crank-Nicolson finite difference method for solving time-fractional diffusion equation, Journal of Fractional Calculus and Applications, 2 (2012) 1-9.

[12] E. Sousa, Finite difference approximations for a fractional advection diffusion problem, Journal of Computational Physics, 228 (2009) 4038-4054.

[13] F. Liu, P. Zhuang, I. Turner, K. Burrage, V. Anh, A new fractional finite volume method for solving the fractional diffusion equation, Applied Mathematical Modelling, 38 (2014) 3871-3878.

[14] H. Hejazi, T. Moroney, F. Liu, Stability and convergence of a finite volume method for the space fractional advectiondispersion equation, Journal of Computational and Applied Mathematics, 255 (2014) 684-697.

[15] A. Elsaid, The variational iteration method for solving Riesz fractional partial differential equations, Computers and Mathematics with Applications, 60 (2010) 1940-1947.

[16] V. Turut, N. Güzel, On solving partial differential equations of fractional order by using the variational iteration method and multivariate Padé approximations, European Journal of Pure and Applied Mathematics, 6 (2013) $147-171$.

[17] E. Sousa, Numerical approximations for fractional diffusion equations via splines, Computers and Mathematics with Applications, 62 (2011) 938-944.

[18] T. S. El-Danaf, A. R. Hadhoud, Parametric spline functions for the solution of the one time fractional Burgers equation, Applied Mathematical Modelling, 36 (2012) 4557-4564.

[19] S. K. Vanani, A. Aminataei, Tau approximate solution of fractional partial differential equations, Computers and Mathematics with Applications, 62 (2011) 1075-1083.

[20] A. Cetinkaya, O. Kiymaz, The solution of the time-fractional diffusion equation by the generalized differential transform method, Mathematical and Computer Modelling, 57 (2013) 2349-2354.

[21] Z. Odibat, S. Momani, A generalized differential transform method for linear partial differential equations of fractional order, Applied Mathematics Letters, 21 (2008) 194-199.

[22] Q. Wang, Numerical solutions for fractional KdV-Burgers equation by Adomian decomposition method, Applied Mathematics and Computation, 182 (2006) 1048-1055.

[23] S. S. Ray, Analytical solution for the space fractional diffusion equation by two-step Adomian decomposition method, Communications in Nonlinear Science and Numerical Simulation, 14 (2009) 1295-1306.

[24] M. Garg, A. Sharma, Solution of space-time fractional telegraph equation by Adomian decomposition method, Journal of Inequalities and Special Functions, 2 (2011) 1-7. 
[25] E. Doha, A. Bhrawy, S. Ezz-Eldien, Efficient Chebyshev spectral methods for solving multi-term fractional orders differential equations, Applied Mathematical Modelling, 35 (2011) 5662-5672.

[26] A. Borhanifar, S. Valizadeh, Numerical solution for fractional partial differential equations using Crank-Nicolson method with Grünwald estimate, Walailak Journal of Science and Technology, 9 (2012) 433-444.

[27] F. Mainardi, R. Gorenflo, On Mittag-Leffler-type functions in fractional evolution processes, Journal of Computational and Applied Mathematics, 118 (2000) 283-299.

[28] M. Yousuf, On the class of high order time stepping schemes based on Padé approximations for the numerical solution of Burgers equation, Applied Mathematics and Computation, 205 (2008) 442-453.

[29] R. L. Burden, J. D. Faires, Numerical Analysis, Brooks/Cole Pub, Boston, 1997.

[30] E. Isaacson, H. Keller, Analysis of Numerical Methods, Wiley, New York, 1966. 